\title{
Predictive value of near-infrared spectroscopy on neurodevelopmental outcome after surgery for congenital heart disease in infancy
}

\author{
Julie Simons, PhD, ${ }^{\mathrm{a}, \mathrm{b}}$ Erica D. Sood, PhD, ${ }^{\mathrm{a}, \mathrm{b}}$ Christopher D. Derby, MD, ${ }^{\mathrm{a}}$ and Christian Pizarro, $\mathrm{MD}^{\mathrm{a}}$
}

Objective: To explore the relationship between intraoperative regional cerebral oxygen saturation $\left(\mathrm{rSO}_{2}\right)$ measured by near-infrared spectroscopy (NIRS) and neurodevelopmental outcome in children after cardiac surgery.

\begin{abstract}
Methods: Cross-sectional neurodevelopmental evaluation at 2 years of age was performed in a cohort of young infants who had surgery for congenital heart disease in 2007. The third edition of Bayley Scales of Infant and Toddler Development (Bayley-III) was used to assess cognitive, language, and motor functioning. Clinical and perioperative data were collected, including intraoperative $\mathrm{rSO}_{2}$ nadir, $\mathrm{rSO}_{2}$ percent decrease from baseline, and cumulative minutes of at least $20 \%, 30 \%$, and $40 \%$ decrease from baseline.
\end{abstract}

Results: Twenty-seven patients without chromosomal abnormality were included in analyses. Mean Bayley-III scores fell within 1 standard deviation of the normative mean. Stepwise regression analyses of patient- and procedure-related variables, including $\mathrm{rSO}_{2}$, demonstrated that cognitive ability was predicted by length of hospital stay and premature birth (58.1\% of variance), receptive communication was predicted by length of hospital stay and $\mathrm{rSO}_{2}$ nadir $(40.2 \%$ of variance), expressive communication was predicted by birth weight $(26.2 \%$ of variance), fine motor functioning was predicted by duration of cardiac intensive care unit stay (41.4\% of variance), and gross motor functioning was predicted by the presence of a significant comorbidity ( $43.5 \%$ of variance).

Conclusions: In a contemporary cohort of infants undergoing surgery for congenital heart disease, neurodevelopmental outcomes at 2 years of age are largely influenced by patient-related characteristics. Although receptive communication appears to be influenced by $\mathrm{rSO}_{2}$ nadir, the predictive value of NIRS remains unclear. (J Thorac Cardiovasc Surg 2012;143:118-25)

With the dramatic decline in mortality among children with congenital heart disease (CHD), attention has been directed toward functional outcomes. Neurodevelopmental impairment is the most common morbidity among survivors with CHD, limiting academic achievement, employability, and overall quality of life. ${ }^{1}$ Intraoperative regional cerebral oxygen saturation $\left(\mathrm{rSO}_{2}\right)$ has been identified as a potential contributor to neurodevelopmental outcomes among children undergoing cardiac surgery. ${ }^{2}$ Monitoring of $\mathrm{rSO}_{2}$ with near-infrared spectroscopy (NIRS), a noninvasive optical technique that monitors brain tissue oxygenation through measurement of oxyhemoglobin and deoxyhemoglobin concentrations, is gaining acceptance. ${ }^{3-5}$ However, technical issues in the measurement of cerebral oxygen,

From the Nemours Cardiac Center, ${ }^{\mathrm{a}}$ and Division of Behavioral Health, ${ }^{\mathrm{b}}$ Alfred I. duPont Hospital for Children, Wilmington, Del.

This project was supported by a grant from the Nemours Foundation (Grant No. 1607700-001).

Disclosures: Authors have nothing to disclose with regard to commercial support.

Read at the 91st Annual Meeting of The American Association for Thoracic Surgery, Philadelphia, Pennsylvania, May 7-11, 2011.

Received for publication May 9, 2011; revisions received July 19, 2011; accepted for publication Sept 15, 2011; available ahead of print Oct 31, 2011.

Address for reprints: Erica Sood, PhD, Nemours Cardiac Center \& Division of Behavioral Health, Alfred I. duPont Hospital for Children, 1600 Rockland Rd, Wilmington, DE 19803 (E-mail: esood@nemours.org).

$0022-5223 / \$ 36.00$

Copyright (C) 2012 by The American Association for Thoracic Surgery doi: 10.1016/j.jtcvs.2011.09.007 methodologic limitations of previous studies, and a lack of evidence correlating NIRS values with neurodevelopmental scores or demonstrating that NIRS data can be used to improve outcomes are commonly cited as obstacles to embracing NIRS monitoring as routine in pediatric cardiac surgery. ${ }^{6}$ Only a few published reports examine the relationship between perioperative oxygenation and later neurodevelopmental functioning, ${ }^{7-9}$ and unfortunately these studies have used inconsistent methods and, commonly, very small sample sizes. Therefore, the existing data cannot provide the necessary confirmation about the usefulness of this tool. This study seeks to examine the predictive value of NIRS by evaluating the relationship between $\mathrm{rSO}_{2}$ data obtained using intraoperative NIRS and neurodevelopmental outcome at 2 years of age.

\section{METHODS}

\section{Participants and Study Design}

The current investigation is a retrospective cross-sectional study examining the relationship between intraoperative $\mathrm{rSO}_{2}$ measured by NIRS and neurodevelopmental outcome in young children with CHD. Participants were 27 children without chromosomal abnormalities who underwent surgery for CHD at our institution in 2007. All patients were under the age of 12 months at the time of the operation and $24 \pm 3$ months at the time of study participation. Patients who were unable to undergo neurodevelopmental testing or were non-English speaking were excluded from 


\section{Abbreviations and Acronyms \\ Bayley-III = Bayley Scales of Infant and Toddler Development, Third Edition \\ CHD = congenital heart disease \\ CICU = cardiac intensive care unit \\ DHCA = deep hypothermic circulatory arrest \\ NIRS = near-infrared spectroscopy \\ PDI $\quad=$ Psychomotor Development Index \\ $\mathrm{rSO}_{2} \quad=$ regional cerebral oxygen saturation}

enrollment. The Nemours Institutional Review Board approved the study, and informed consent and parental permission were obtained from the parents or legal guardians before study participation.

A total of 79 patients under the age of 12 months underwent open cardiac operations for a cardiac defect using NIRS monitoring in 2007. There were 62 English-speaking survivors at the time of study enrollment, and 43 of their families provided informed consent. Nineteen families did not consent owing to factors such as family relocation or lack of interest. Of those who did consent, 39 were tested. Twelve of the tested patients had a documented chromosomal abnormality and were excluded from the present analyses, resulting in a final sample size of 27.

\section{Operative Management}

Patients were managed preoperatively and postoperatively in a dedicated cardiac intensive care unit (CICU). Perfusion management included $\mathrm{pH}$-stat strategy during cooling with a target hematocrit value of $30 \%$, as well as fresh whole blood and a single dose of methylprednisolone in the cardiopulmonary bypass prime. Deep hypothermic circulatory arrest (DHCA) was used at the discretion of the surgeon. No variation in perfusion practices or personnel occurred during the study period. Perioperative management decisions were not made on the basis of NIRS data.

\section{NIRS}

Regional cerebral oxygenation with NIRS (INVOS 5100B; Somanetics, Troy, Mich) was routinely recorded during surgery for CHD by placement of a probe on the forehead in the midline position. The INVOS is a continuous-wave near-infrared spectrometer that uses 2 wavelengths of light (730 and $810 \mathrm{~nm}$ ) to measure the ratio of oxyhemoglobin to total hemoglobin to derive oxygen saturation, the scale unit of which is percent. This information was then used to calculate (1) the lowest saturation level during the operative period (nadir), (2) the percent decrease from baseline to nadir, (3) $\mathrm{rSO}_{2}$ nadir below $40 \%, 30 \%$, and $20 \%$ (yes/no variables), (4) $20 \%$, $30 \%$, and $40 \%$ decrease from baseline (yes/no variables), and (5) cumulative minutes at $20 \%, 30 \%$, and $40 \%$ decrease from baseline. We examined both absolute and relative measures of $\mathrm{rSO}_{2}$. In addition to comparing the neurodevelopmental outcomes of patients above and below the levels suggested to be associated with increased risk $\left(\mathrm{rSO}_{2}\right.$ of $40 \%$ or $20 \%$ decrease from baseline), ${ }^{5}$ comparison of patients falling above and below alternative levels was performed given the lack of empirical data correlating any specific level with neurodevelopment outcomes. NIRS data were unavailable for 1 participant; therefore, sample size was 26 for regression analyses involving NIRS variables.

\section{Neurodevelopmental Testing}

Children were administered the Bayley Scales of Infant and Toddler Development, Third Edition (Bayley-III) ${ }^{10}$ by a clinical psychologist according to standardization. The Bayley-III assesses the developmental functioning of infants and young children between the ages of 1 month and 42 months through items administered to the child in a structured play format. As compared with the Bayley-II, this updated measure allows for the calculation of separate cognitive, receptive communication, and expressive communication scaled scores (previously combined into the Bayley-II Mental Development Index) and separate fine motor and gross motor scaled scores (previously combined into the Bayley-II Psychomotor Development Index [PDI]). Scaled scores have a mean of 10 and a standard deviation of 3. After the evaluation, families who expressed interest in obtaining feedback regarding the results of their child's evaluation were provided with a brief verbal debriefing. We were able to obtain only partial neurodevelopmental data for 7 of the 27 patients owing to child fussiness or refusal to complete testing. All available data for these patients were included in analyses.

\section{Analytic Strategy}

Exploratory univariable analyses assessed the relationships of patientand procedure-related characteristics and NIRS variables to neurodevelopmental scores. Procedure-related characteristics referred to the operation with NIRS monitoring in 2007, with the exception of history of multiple cardiac procedures, history of multiple procedures with DHCA, and cumulative exposure to DHCA. Associations with sociodemographic variables were also examined. For multivariable analysis, only those variables associated with each neurodevelopmental outcome at $P<.05$ were considered in forward stepwise regression analyses. The entry criterion was set at $P<.05$ and the removal criterion was set at $P \geq .10$. Explained variance for all models was calculated by adjusted $R^{2}$ values.

To further examine the predictive value of NIRS on neurodevelopmental outcome, we performed secondary analyses in which all patients were stratified into 2 groups for each neurodevelopmental domain: delayed and nondelayed. Patients were considered delayed if their score was more than 1 standard deviation below the normative mean. All other patients were considered nondelayed. Independent samples $t$ test (for continuous variables) and Fisher's exact test (for dichotomous variables) were used to compare the 2 groups.

The Nemours Foundation was not involved in data interpretation.

\section{RESULTS \\ Baseline Characteristics}

Cardiac procedures for the 27 patients included in analyses were Fontan or hemi-Fontan $(n=6)$, arterial switch $(n=3)$, Ross-Konno $(n=2)$, Norwood $(n=2)$, orthotopic heart transplantation $(\mathrm{n}=2)$, and repair of a ventricular septal defect $(\mathrm{n}=5)$, right ventricular outflow tract obstruction $(\mathrm{n}=3)$, tetralogy of Fallot $(\mathrm{n}=2)$, truncus $(\mathrm{n}=1)$, and coarctation/ventricular septal defect $(\mathrm{n}=1)$. Table 1 reports patient- and procedure-related characteristics for these 27 patients and for the 16 eligible patients without chromosomal abnormalities who did not enroll or participate in neurodevelopmental testing. Table 2 reports sociodemographic information for the study sample. Patients who did not enroll or participate in testing were more likely to be of an ethnic minority group than those who did participate in testing ( $56 \%$ vs $26 \% ; P=.047)$. Table 3 reports descriptive data for the NIRS variables in this sample.

\section{Neurodevelopmental Outcomes}

Receptive communication (9.2 \pm 3.5$)$, expressive communication $(9.5 \pm 3.4)$, and fine motor $(9.3 \pm 3)$ development were comparable with that of the general 
TABLE 1. Patient-and procedure-related characteristics of tested and nontested patients

\begin{tabular}{|c|c|c|}
\hline & $\begin{array}{c}\text { Tested } \\
(\mathbf{n}=\mathbf{2 7})\end{array}$ & $\begin{array}{c}\text { Nontested } \\
(\mathrm{n}=16)\end{array}$ \\
\hline & \multicolumn{2}{|c|}{ Median (1Q-3Q) } \\
\hline Birth weight $(\mathrm{kg})$ & $3.2(2.6-3.3)$ & $3.2(2.3-3.5)$ \\
\hline Age at operation (mo) & $1.9(1.0-5.0) \dagger$ & $4.5(1.5-7.6)$ \\
\hline Weight at operation $(\mathrm{kg})$ & $4.5(3.5-5.8) \dagger$ & $5.6(4.0-7.3)$ \\
\hline Aristotle Complexity Score & $11.5(8.0-14.3)$ & $9.9(6.3-13.6)$ \\
\hline Total CPB support (min) & $82.5(73-121)$ & $80(55.5-124.0)$ \\
\hline DHCA (min) & $39(0-43)$ & $38.5(0-40.8)$ \\
\hline CICU stay (d) & $6(2-15)$ & $2.5(1-12.0)$ \\
\hline Hospital stay (d) & $11(8-22)$ & $12.5(6.3-65.3)$ \\
\hline \multirow[t]{2}{*}{$\begin{array}{l}\text { Cumulative exposure to } \\
\text { DHCA (min) }\end{array}$} & $42(38-85)$ & $39.5(3.8-62.8)$ \\
\hline & \multicolumn{2}{|c|}{ Frequency $(\%)$} \\
\hline Premature birth $(<37$ wk) & $3(11.1 \%)$ & $2(12.5 \%)$ \\
\hline Significant comorbidity* & $9(33.3 \%)$ & $5(31.3 \%)$ \\
\hline Single-ventricle physiology & $8(29.6 \%)$ & $3(18.8 \%)$ \\
\hline Preoperative CICU & $10(37.0 \%)$ & $7(43.8 \%)$ \\
\hline Use of DHCA & $19(70.4 \%)$ & $11(68.8 \%)$ \\
\hline Preoperative cyanosis & $12(44.4 \%)$ & $8(50.0 \%)$ \\
\hline Multiple procedures (lifetime) & $12(44.4 \%)$ & $9(56.3 \%)$ \\
\hline $\begin{array}{l}\text { Multiple procedures with } \\
\text { DHCA (lifetime) }\end{array}$ & $10(37.0 \%)$ & $3(18.8 \%)$ \\
\hline \multicolumn{3}{|c|}{$\begin{array}{l}1 Q \text {, First quartile; } 3 Q \text {, third quartile; } C P B \text {, cardiopulmonary bypass; } D H C A \text {, deep hy- } \\
\text { pothermic circulatory arrest; } C I C U \text {, cardiac intensive care unit. *Significant comor- } \\
\text { bidities of tested participants included restrictive atrial septal defect, prematurity } \\
\text { ( }<35 \text { weeks' gestational age), arrhythmia, necrotizing enterocolitis, seizures, de- } \\
\text { pressed myocardial function, history of prolonged mechanical ventilation/portal hy- } \\
\text { pertension/pneumonia, protein-losing enteropathy/right ventricle dysfunction/ } \\
\text { pacemaker. } \dagger P<.10 \text {. }\end{array}$} \\
\hline
\end{tabular}

population. Mean cognitive $(8.2 \pm 3.3)$ and gross motor $(8.1$ \pm 1.8 ) scores were significantly lower than published norms $(P<.05)$, although still within 1 standard deviation of the normative mean $(10 \pm 3)$. Figure 1 displays neurodevelopmental outcomes for the total sample, participants with $\mathrm{rSO}_{2}$ nadir of $40 \%$ or more $(\mathrm{n}=21)$, and participants with $\mathrm{rSO}_{2}$ nadir less than $40 \%(\mathrm{n}=5)$.

\section{Predictors of Bayley-III Scores}

Sociodemographic variables were not associated with any of the neurodevelopmental domains in exploratory univariable analyses and were therefore not subsequently considered in stepwise regression analyses. Neither preoperative cyanosis nor postoperative cyanosis was significantly associated with any of the neurodevelopmental domains in exploratory univariable analyses, therefore not meeting entry criterion for multivariable stepwise regression analyses. When postoperative cyanosis was forced into the regression models, it continued to have nonsignificant relationships with all 5 neurodevelopmental domains and did not affect which variables were included in each final multivariable model. Table 4 reports final stepwise regression models for each neurodevelopmental outcome.
TABLE 2. Sociodemographic variables for the study sample $(n=27)$

\begin{tabular}{lc}
\hline \multicolumn{1}{c}{ Variable } & Frequency $(\%)$ \\
\hline Gender & \\
Male & $14(51.9 \%)$ \\
Female & $13(48.1 \%)$ \\
Race/ethnicity & \\
Nonhispanic white & $20(74.1 \%)$ \\
Black or African American & $5(18.5 \%)$ \\
Hispanic or latino & $1(3.7 \%)$ \\
Asian American & $1(3.7 \%)$ \\
Household income & \\
$<\$ 39,999$ & $10(37.0 \%)$ \\
\$40,000-\$69,999 & $7(25.9 \%)$ \\
\$70,000-\$99,999 & $7(25.9 \%)$ \\
$>\$ 100,000$ & $3(11.1 \%)$ \\
Education level of accompanying parent & \\
High school/GED/vocational & $8(29.6 \%)$ \\
Some college & $7(25.9 \%)$ \\
College degree & $8(29.6 \%)$ \\
Graduate degree & $4(14.8 \%)$ \\
Education level of spouse/partner $(\mathrm{n}=22)$ & \\
High school/GED/vocational & $6(27.3 \%)$ \\
Some college & $8(36.4 \%)$ \\
College degree & $6(27.3 \%)$ \\
Graduate degree & $2(9.1 \%)$ \\
\hline$G E D$, General Educational Development &
\end{tabular}

GED, General Educational Development.

Cognitive. Premature birth, single-ventricle physiology, presence of a significant comorbidity, age at operation, length of stay in the pediatric CICU, length of hospital stay, and history of multiple procedures with DHCA were correlated with cognitive scores in univariable analyses. None of the NIRS variables studied was associated with cognitive scores. The final multivariable model included length of hospital stay and premature birth and accounted for $58.1 \%$ of the variability in cognitive outcome.

TABLE 3. NIRS variables $(n=26)$

\begin{tabular}{lc}
\hline Continuous variables & Median (1Q-3Q) \\
$\mathrm{rSO}_{2}$ nadir & $42.5(40-49)$ \\
$\mathrm{rSO}_{2} \%$ decrease from baseline & $32.7 \%(22.9-46.6 \%)$ \\
Minutes at $20 \%$ decrease from baseline & $9.0(2.5-38.5)$ \\
Minutes at $30 \%$ decrease from baseline & $1.0(0-29.5)$ \\
Minutes at $40 \%$ decrease from baseline & $0(0-8.0)$ \\
Categorical variables & Frequency $(\%)$ \\
$\mathrm{rSO}_{2}$ nadir $<40 \%$ & $5(19.2 \%)$ \\
$\mathrm{rSO}_{2}$ nadir $<30 \%$ & $4(15.4 \%)$ \\
$\mathrm{rSO}_{2}$ nadir $<20 \%$ & $3(11.5 \%)$ \\
$\mathrm{Percentage} \mathrm{decrease} \mathrm{from} \mathrm{baseline}_{20 \%}$ & \\
$30 \%$ & $22(84.6 \%)$ \\
$40 \%$ & $14(53.8 \%)$ \\
\hline
\end{tabular}

NIRS, Near-infrared spectroscopy; $1 Q$, First quartile; $3 Q$, third quartile; $r \mathrm{SO}_{2}$, intraoperative regional cerebral oxygen saturation. 


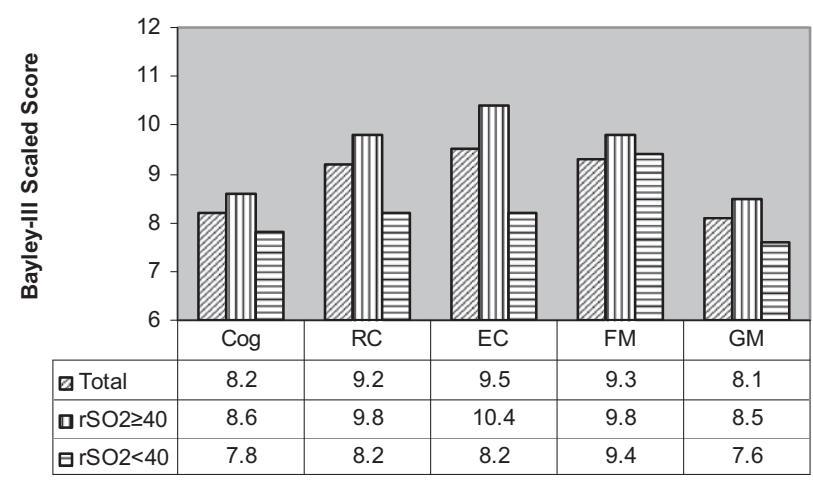

FIGURE 1. Neurodevelopmental outcomes of total sample $(\mathrm{n}=27), \mathrm{rSO}_{2}$ nadir $\geq 40(\mathrm{n}=21)$, and $\mathrm{rSO}_{2}$ nadir $<40(\mathrm{~N}=5)$. Note. Scores have a normative mean of 10 and standard deviation of 3. $\operatorname{Cog}$, Cognitive; $R C$, receptive communication; $E C$, expressive communication; $F M$, fine motor; $G M$, gross motor.

Receptive communication. Premature birth, presence of a significant comorbidity, $\mathrm{rSO}_{2}$ nadir, and length of hospital stay were correlated with receptive communication scores in univariable analyses. The final multivariable model included length of hospital stay and $\mathrm{rSO}_{2}$ nadir and accounted for $40.2 \%$ of the variability in receptive communication outcome.

Expressive communication. Birth weight and presence of a significant comorbidity were correlated with expressive communication scores in univariable analyses. None of the NIRS variables studied was associated with expressive communication. The final multivariable model included birth weight and accounted for $26.2 \%$ of the variability in expressive communication outcome.

Fine motor. Premature birth, presence of a significant comorbidity, a $20 \%$ decrease in $\mathrm{rSO}_{2}$ from baseline (yes/no variable), duration of stay in the CICU, and length of hospital stay were correlated with motor scores in univariable analyses. The final multivariable model included duration of stay in the CICU and accounted for $41.4 \%$ of the variability in fine motor outcome.

Gross motor. Premature birth, presence of a significant comorbidity, history of multiple cardiac procedures, and cumulative exposure to DHCA were correlated with gross motor scores at $P<.05$ in univariable analyses. None of the NIRS variables studied was associated with gross motor scores. The final multivariable model included presence of a significant comorbidity and accounted for $43.5 \%$ of the variability in gross motor outcome.

\section{Predictors of Developmental Delay}

Patients were categorized as delayed ( $>1$ standard deviation below the normative mean) versus nondelayed with regard to cognitive, receptive communication, expressive communication, fine motor, and gross motor functioning.

Cognitive. Patients who exhibited delayed cognitive abilities were more likely to have a significant comorbidity (4/5;
TABLE 4. Final stepwise regression models

\begin{tabular}{lccccc}
\hline $\begin{array}{c}\text { Neurodevelopmental } \\
\text { outcomes }\end{array}$ & $\boldsymbol{B}$ & $\boldsymbol{S E}(\boldsymbol{B})$ & $\boldsymbol{\beta}$ & $\boldsymbol{P}$ & $\boldsymbol{R}^{\mathbf{2}}$ \\
\hline Regression 1: Cognitive & & & & & 0.58 \\
$\quad$ Length of hospital stay & -.05 & .01 & -.67 & $<.001$ & \\
$\quad$ Premature birth & -5.0 & 1.3 & -.48 & .001 & \\
$\quad \begin{array}{l}\text { Regression 2: Receptive } \\
\quad \text { communication }\end{array}$ & & & & & 0.40 \\
$\quad \begin{array}{l}\text { Length of hospital stay } \\
\text { rSO } \text { nadir }\end{array}$ & -.03 & .01 & -.53 & .004 & \\
Regression 3: Expressive & .11 & .04 & .44 & .01 & \\
$\quad$ communication & & & & & 0.26 \\
$\quad$ Birth weight & 4.1 & 1.6 & .56 & .02 & \\
Regression 4: Fine motor & & & & & 0.41 \\
$\quad$ Duration of CICU stay & -.05 & .01 & -.67 & .001 & \\
Regression 5: Gross motor & & & & & 0.44 \\
$\quad$ Significant comorbidity & -2.5 & .63 & -.68 & .001 & \\
\hline
\end{tabular}

$\mathrm{SE}$, Standard error; $r \mathrm{SO}_{2}$, intraoperative regional cerebral oxygen saturation; $\mathrm{CICU}$, cardiac intensive care unit.

$80 \%)$ as compared with those without cognitive delays $(5 / 22 ; 23 \%)(P=.03$; Fisher's exact test $)$ and were more likely to have multiple procedures with DHCA (4/5; $80 \%)$ as compared with those without cognitive delays $(6 / 22 ; 27 \%)(P=.047$; Fisher's exact test $)$.

Receptive communication. Patients who exhibited delayed receptive communication had an older age at operation as compared with those without receptive communication delays $\left(\mathrm{t}_{23}=2.2 ; P=.04\right)$, and were more likely to have a significant comorbidity $(4 / 5 ; 80 \%)$ as compared with those without receptive communication delays $(5 / 20 ; 25 \%)(P=.04$; Fisher's exact test $)$.

Expressive communication. Patients who exhibited delayed expressive communication were more likely to have a significant comorbidity $(4 / 4 ; 100 \%)$ as compared with those without expressive communication delays (4/ $18 ; 22 \%)(P=.01$; Fisher's exact test $)$ and were more likely to have an $\mathrm{rSO}_{2}$ nadir of less than $20 \%$ saturation $(2 / 3 ; 67 \%)$ as compared with those without expressive communication delays $(1 / 18 ; 6 \%)(P=.04$; Fisher's exact test).

Fine motor. Patients who exhibited delayed fine motor abilities were more likely to have been born prematurely $(2 / 3 ; 67 \%)$ as compared with those without fine motor delays $(1 / 19 ; 5 \%)(P=.04$; Fisher's exact test $)$ and were more likely to have a significant comorbidity $(3 / 3 ; 100 \%)$ as compared with those without fine motor delays $(5 / 19$; $26 \%)(P=.04$; Fisher's exact test $)$.

Gross motor. Patients who exhibited delayed gross motor abilities had a longer duration of DHCA as compared with those without gross motor delays $\left(\mathrm{t}_{18}=2.6\right.$; $P=.02)$ and were more likely to have a significant comorbidity $(4 / 4 ; 100 \%)$ as compared with those without gross motor delays $(3 / 16 ; 19 \%)(P=.007$; Fisher's exact test). 


\section{DISCUSSION}

Intraoperative $\mathrm{rSO}_{2}$ measured by NIRS was not a robust predictor of neurodevelopmental outcomes in this sample after accounting for patient- and procedure-related variables. Receptive communication, expressive communication, and fine motor performance were each associated with a measure of $\mathrm{rSO}_{2}$ in univariable analyses; however, only the relationship between $\mathrm{rSO}_{2}$ nadir and receptive communication remained significant in multivariable analyses. These results suggest that a given $\mathrm{rSO}_{2}$ may not have the same impact on all neurodevelopmental domains. Whereas intraoperative $\mathrm{rSO}_{2}$ as measured by NIRS may be a useful predictor of later language abilities, it does not appear to predict cognitive or motor sequelae at 2 years of age. It should be noted that less variability across patients was observed on gross motor tasks as compared with other Bayley-III domains and the normative sample, and as a group, patients scored at the lower end of the normal range on the gross motor domain. This domain may be more universally affected in children with CHD who have undergone cardiac surgery. Alternatively, given that the gross motor subtest is typically administered last, poor performance on this subtest may be a surrogate for the attentional difficulties that have been previously documented in children with CHD. ${ }^{11}$

Despite the frequent use of DHCA in this patient cohort, few children dropped below $40 \% \mathrm{rSO}_{2}$. This is consistent with other recent studies examining intraoperative $\mathrm{rSO}_{2}{ }^{8}$ The higher intraoperative $\mathrm{rSO}_{2}$ observed in this study could be explained by the practice of limiting DHCA to a duration of under 40 minutes whenever possible and using intermittent reperfusion. It is possible that longer periods of DHCA, associated with prolonged and more severe drops in $\mathrm{rSO}_{2}$, may have revealed additional relationships between $\mathrm{rSO}_{2}$ and neurodevelopmental outcome.

The results of this study differ in some important ways from the existing literature on $\mathrm{rSO}_{2}$ and neurodevelopmental outcome. Whereas we found that $\mathrm{rSO}_{2}$ was related to later language development, Kussman and associates ${ }^{8}$ reported a relationship between $\mathrm{rSO}_{2}$ and the Bayley-II PDI only. Similarly, although Toet and colleagues ${ }^{9}$ did not find a significant difference in neurodevelopmental outcome above and below preoperative $\mathrm{rSO}_{2}$ values of $35 \%$, a review of the Bayley-II mean scores suggests that if there were a difference, it would likely be specific to PDI. Although Hoffman and associates ${ }^{7}$ reported a relationship between low systemic mixed venous oxygen levels and neurodevelopmental outcomes in children undergoing cardiac surgery, there was no direct relationship with NIRS data; poor neurodevelopmental outcomes in their patients may have been related to globally poor perfusion that may or may not be evident in NIRS monitoring of $\mathrm{rSO} 2$.

Heretofore, studies of NIRS and neurodevelopmental outcomes have been limited by the absence of other important patient-related variables known to affect outcome. $^{1,12}$ For example, whereas Kussman and associates ${ }^{8}$ did adjust for age 30 days or less, diagnosis group, and social class, they did not consider other potentially important variables such as premature birth, birth weight, and significant medical comorbidities. Without the inclusion of these potential confounding variables, it is not clear whether $\mathrm{rSO}_{2}$ is an independent predictor of PDI scores.

Given a small number of studies directly examining neurodevelopmental outcomes in relation to perioperative oxygenation and varying methodologies, firm conclusions cannot yet be drawn about the utility of $\mathrm{rSO}_{2}$ monitoring for patients undergoing cardiac surgery. In addition, the studies conducted thus far differ with regard to the neurodevelopmental testing battery, age at which children were tested, underlying cardiac diagnoses of the study sample, technology used to assess oxygenation, perioperative phase during which $\mathrm{rSO}_{2}$ data were collected (preoperative, intraoperative, or postoperative), and analytic techniques. The ways in which $\mathrm{rSO}_{2}$ was measured and defined also differ across studies and have included average or nadir $\mathrm{rSO}_{2}$, desaturation below a specified level, and integrated $\mathrm{rSO}_{2}$ (minutes $\times$ desaturation points below a specified level). To complicate matters further, the specified $\mathrm{rSO}_{2}$ level has not been consistent across studies and, in some cases, has represented the median sample $\mathrm{rSO}_{2}$ rather than a cut point that optimally differentiates between children with and without delays. It will be important for future studies to identify the $\mathrm{rSO}_{2}$ threshold that may lead to neurodevelopmental delays and confirm the predictive value of this monitoring tool in relation to specific domains.

Our data suggest that in a cohort of children undergoing surgery for congenital heart defects during infancy, patient-related characteristics, such as premature birth and significant comorbidities, appear to be more robust predictors of neurodevelopment than intraoperative $\mathrm{rSO}_{2}$. Patient-related characteristics were also implicated when patients exhibiting delay were compared with those without delay on each neurodevelopmental domain. These findings are consistent with prior research suggesting that characteristics inherent to the child, present at birth, are often important contributors to neurodevelopmental delay. ${ }^{1,12}$ Also, as previously reported,${ }^{13}$ length of stay in the hospital and the CICU were associated with neurodevelopmental outcomes, even after accounting for the effects of potentially confounding patient- and procedure-related characteristics. Length of stay is likely a surrogate variable reflecting the influences of other factors affecting the central nervous system and neurodevelopment. Young children who experience a prolonged hospital stay are likely sicker, prone to complications, and may be missing important experiences and opportunities that would promote 
their early development. Further research is necessary to determine the mechanisms underlying this relation.

Limitations of this study include its small sample size and the limited statistical power to detect true differences and relationships. Given the interrelatedness of patient- and procedure-related characteristics, large samples are needed to determine which variables are driving observed effects. Despite the frequent use of DHCA in this patient cohort, few children had an $\mathrm{rSO}_{2}$ below 40 or a decrease from baseline of $40 \%$ or more, resulting in limited statistical power to compare children above and below the specified levels. Although we had planned to also calculate cumulative minutes below $40 \%, 30 \%$, and $20 \%$ saturation, these variables were eliminated owing to the low number of participants whose $\mathrm{rSO}_{2}$ dropped below the specified levels. It should be noted that almost half of the participants in this study had multiple operations and hospitalizations; therefore, whereas the primary focus of this study was on the specific operation during which the patient received NIRS monitoring, it is possible that events occurring during other operative procedures affected neurodevelopmental outcomes at 24 months of age. In addition, although neurodevelopmental outcomes could be influenced by events at any time in the perioperative period, NIRS values were only monitored during the surgical procedure. Therefore, important events that may have played a role in neurodevelopmental outcomes could have gone undetected.

In conclusion, despite the intuitive appeal of $\mathrm{rSO}_{2}$ monitoring by NIRS during cardiac surgery, the predictive value of NIRS for neurodevelopmental outcome remains unclear. As suggested by these preliminary data, the predictive value of NIRS may vary for different neurodevelopmental domains, and it seems likely that this type of monitoring may prove to be most useful in patients subjected to cyanosis, hemodynamic instability, and repeated interventions, a population commonly perceived to be at highest risk for neurodevelopmental impairment.

We thank Mrs Carol Prospero for her assistance in coordinating this study.

\section{References}

1. Wernovsky G. Current insights regarding neurological and developmental abnormalities in children and young adults with complex congenital cardiac disease. Cardiol Young. 2006;16(1 suppl):92-104.

2. Daubeney PEF, Smith DC, Pilkington SN, Janke E, Gareth AC, Smith DC, et al. Cerebral oxygenation during paediatric cardiac surgery: identification of vulnerable periods using near infrared spectroscopy. Eur J Cardiothoracic Surg. 1998; 13:370-7.

3. Edmonds HL. Pro: All cardiac surgical patients should have intraoperative cerebral oxygenation monitoring. J Cardiothorac Vasc Anesth. 2006;20:445-9.

4. Hoffman GM. Neurologic monitoring on cardiopulmonary bypass: what are we obligated to do? Ann Thorac Surg. 2006;81:S2373-80.

5. Hoffman GM. Pro: Near-infrared spectroscopy should be used for all cardiopulmonary bypass. J Cardiothorac Vasc Anesth. 2006;20:606-12.

6. Davies LK, Janelle GM. Con: All cardiac surgical patients should not have intraoperative cerebral oxygenation monitoring. J Cardiothorac Vasc Anesth. 2006; 20:450-5.
7. Hoffman GM, Mussatto KA, Brosig CL, Ghanayem NS, Musa N, Fedderly RT, et al. Systemic venous oxygen saturation after the Norwood procedure and childhood neurodevelopmental outcome. J Thorac Cardiovasc Surg. 2005;130: 1094-100.

8. Kussman BD, Wypij D, Laussen PC, Soul JS, Bellinger DC, DiNardo JA, et al. Relationship of intraoperative cerebral oxygen saturation to neurodevelopmental outcome and brain magnetic resonance imaging at 1 year of age in infants undergoing biventricular repair. Circulation. 2010;122:245-54

9. Toet MC, Flinterman A, van de Laar I, Vries JW, Bennink GB, Uiterwaal CS, et al. Cerebral oxygen saturation and electrical brain activity before, during, and up to 36 hours after arterial switch procedure in neonates without preexisting brain damage: its relationship to neurodevelopmental outcome. Exp Brain Res. 2005; 165:343-50

10. Bayley N. Bayley scales of infant development. 3rd ed. San Antonio (TX): The Psychological Corporation; 2006.

11. Bellinger DC, Wypij D, duPlessis AJ, Rappaport LA, Jonas RA, Wernovsky G, et al. Neurodevelopmental status at eight years in children with dextrotransposition of the great arteries: the Boston Circulatory Arrest Trial. J Thorac Cardiovasc Surg. 2003;126:1385-96.

12. Gaynor JW, Wernovsky G, Jarvik GP, Bernbaum J, Gerdes M, Zackai E, et al. Patient characteristics are important determinants of neurodevelopmental outcome at one year of age after neonatal and infant cardiac surgery. J Thorac Cardiovasc Surg. 2007;133:1344-53.

13. Newburger JW, Wypij D, Bellinger DC, du Plessis AJ, Kuban KC, Rappaport LA, et al. Length of stay after infant heart surgery is related to cognitive outcome at age 8 years. J Pediatr. 2003;143:67-73.

\section{Discussion}

Dr James S. Tweddell (Milwaukee, Wis). My thanks to the authors for sending me their manuscript well in advance of this meeting.

The authors have performed a prospective observational study of infants less than 1 year of age looking for factors predicting neurodevelopmental outcome and have included intraoperative NIRS among those factors tested. They have found that the Bayley scores were predicted by a large number of factors such as hospital length of stay, prematurity, birth weight, length of stay in the cardiac intensive care unit, and comorbidities. Only in the area of receptive communication was the cerebral saturation found to be predictive Just for everyone's benefit, could you explain to us what that particular domain, receptive communication, measures and its prognostic importance?

Dr Sood. Absolutely. It measures receptive language-how much language the child understands. Unlike expressive communication, we are not looking at what the child can actually communicate but instead what he or she understands. For example, a child looks at a book of pictures and is asked to point to the ball, the toy, the bear. Also, the child is observed interacting with the caregiver and the examiner to see how well he or she responds. And your next question refers to the predictive value?

Dr Tweddell. The prognostic significance.

Dr Sood. What we know is that, at 2 years of age, Bayley scores do not correlate highly with later intelligence for most children. The Bayley-III is a measure of development, rather than intelligence. Intelligence is thought to be stable, whereas development is characterized by spurts and lags. However, for children who have known deficits, children who are below the average range, the predictive value tends to be better. For these children, the Bayley is more predictive of impairment later on in childhood and in adulthood.

Dr Tweddell. Thank you. I want to emphasize here that the authors have looked only at the intraoperative NIRS. The basic 
premise of use of NIRS, specifically at our institution, is to minimize or prevent cerebral ischemia that could result in neurologic impairment. Certainly the operating room is one of those arenas where that could occur, but it could also occur in other places, in the case of neonates in the newborn nursery, and for all the patients in the postoperative care unit. Patients can have a decrease in cerebral oxygenation at any time during the hospitalization, and any of those episodes could affect their neurodevelopmental outcome. Particularly in this study, you identified events outside the operating room, such as length of stay in the cardiac intensive care unit and total hospital length of stay, as important factors. Your study suggests that there might be other times when the patient is vulnerable.

Dr Sood. Absolutely.

Dr Tweddell. You also have included a heterogeneous population, and this may be a strength or a weakness. In previous studies in which NIRS was found to more strongly predict neurodevelopmental outcome, the patient populations studied were more uniform than in your study. In our own studies, we have included primarily neonates with a single diagnosis, hypoplastic left heart syndrome. In the recent publication from Boston, the study population comprised patients undergoing 2-ventricle repair. In both cases the study groups were fairly uniform, and perhaps that improves the likelihood of identifying an impact of NIRS by minimizing the impact of confounding factors such as arterial saturation and perfusion temperature.

How is the $\mathrm{rSO}_{2}$ nadir determined? Was that the lowest value?

Dr Sood. Yes, it was the lowest value during surgery.

Dr Tweddell. It was not the inflection point of the decay slope or anything like that?

Dr Sood. Correct.

Dr Tweddell. Okay. How long did you have to stay at that level before it became your nadir?

Dr Sood. You just had to reach that level.

Dr Tweddell. All right. Thank you. What constituted significant comorbidities? Were there any neurologic injuries in that group?

Dr Sood. Comorbidities included restrictive atrial septal defect, prematurity less than 35 weeks' gestational age, arrhythmias, necrotizing enterocolitis, seizures, depressed myocardial function, history of prolonged mechanical ventilation, portal hypertension, pneumonia, protein-losing enteropathy, right ventricular dysfunction, and pacemaker.

Dr Tweddell. Thank you. The seizures in particular could be a neurodevelopmental outcome end point, too, couldn't they? You included both cyanotic and acyanotic children in your study, and $\mathrm{rSO}_{2}$ is related to arterial saturation. How did you control or account for that?

Dr Sood. We included preoperative cyanosis in univariable analyses, and then we looked at what entered into stepwise regression analyses.

Dr Tweddell. Was that an anatomic diagnosis that determined whether the child was cyanotic or was that a measure of arterial saturation that determined cyanosis?

Dr Sood. That was a measure.

Dr Tweddell. Certainly if a child is profoundly cyanotic, the $\mathrm{rSO}_{2}$ is going to be low as well, right? So it might be important to control for that. I can certainly understand the drop to less than $40 \%$ and the lowest value affecting neurodevelopmental outcome, but you found that a decrease to less than $20 \%$ of baseline did not correlate with worse neurodevelopmental outcome. Were those patients different in some way? Did they start off at a higher baseline? Did you consider looking at or controlling for baseline NIRS and how that correlated with outcome?

Dr Sood. This study did not focus on the baseline $\mathrm{rSO}_{2}$, but that is certainly an area of future research.

Dr Tweddell. An $\mathrm{rSO}_{2}$ of $40 \%$ is a pretty widely accepted threshold for cerebral ischemia at normothermia, but at deep hypothermia it may not indicate adequate cerebral oxygenation. The majority of your patients had DHCA. Did you do anything to control for temperature?

Dr Sood. We did not.

Dr Tweddell. The strengths of this paper include the measurement of the potential benefit of NIRS as it is used in the typical clinical situation with a broad spectrum of patients. Ultimately, I think this analysis could benefit from the inclusion of NIRS data from the entire hospitalization and controlling for differences in baseline values, preoperative and postoperative cyanosis, and the impact of deep hypothermia. This will be an important contribution and I thank you for allowing me to discuss it.

Dr Sood. Thank you.

Dr Pizarro. Bill, I just want to offer a couple explanations about the methodology. One of the reasons we chose not to include a specific patient population was that we wanted to try to discern, if at all possible, what is the real contribution of NIRS into our decision making about how we conduct our clinical care in a real world. I think that NIRS is being used in a number of institutions across the board, not for monitoring of a specific subpatient population, but rather for everyone out there. So I think that the fundamental question was: How much do we learn from this insofar that we could correlate with outcomes at least at 2 years of age? We chose to use a most updated version of the Bayley scales that will give us more domains to be tested and then try to discern whether we could predict outcomes without making decisions based on NIRS. I think the lesson is that, perhaps, if we would extend the limits of, for example, circulatory arrest, there would be a far greater yield in trying to make predictions. For example, 100 minutes of circulatory arrest will definitely go along with a very low value of NIRS and likely there will be a bad outcome. But given what the current clinical practice is, we tried to learn what the additional predictive value of NIRS is within the context of standardized practice nowadays.

Dr J. William Gaynor (Philadelphia, Pa). I have a question concerning management. Has this changed your practice? Do you still record intraoperative NIRS? Do you act on intraoperative NIRS? If not, why do you record it? Do you record NIRS in the postoperative period and do you manage patients on the basis of the postoperative NIRS? Has this changed your practice or what you do at all?

Dr Pizarro. I will answer that. The answer is no, yes, no, and no. That is, we continue to record as part of an ongoing study. I think that we walked away from this learning and reaffirming much of what your center and others have put forward, which is the notion that there are many patient variables that really determine what the patients will look like when it comes to neurodevelopmental testing later on. We are still accruing patients, including intensive care unit stay NIRS, to have a far more in-depth analysis. 
Dr Tweddell. Are you going to add postoperative data collection?

Dr Pizarro. Yes, we are doing it.

Dr Tweddell. Thank you.

Dr Constantine Mavroudis (Cleveland, Ohio). Was your question intimating that this is a nice technology but maybe people do not pay attention to it?

Dr Gaynor. I do not want to be the focus of the discussion.

Dr Mavroudis. But you already have been.

Dr Gaynor. There was a recent paper from Dean Andropoulos at Texas Children's where they used a very detailed neuromonitoring program with transcranial Doppler sonography and NIRS intraoperatively, and they have a very detailed protocol to interact, to respond to those changes. They have looked at preoperative and postoperative brain magnetic resonance imaging. The most important predictor of new injury was brain immaturity. None of the NIRS variables that they measured correlated with outcome. So my question is, will some centers actually use that number? How is that number being used? If it is being recorded as part of a research study, that's one thing. If it is being recorded clinically but not being acted on, that's a different question.

Dr Mavroudis. My impression, and this is going to be anecdotal information on the basis of a worldwide survey, is that those who use it will use it in the operating room. More than once I have had a catheter in the wrong place, the anesthesiologist has alerted me that the NIRS had fallen off, and I have adjusted the catheter. I think we saved a significant problem there. So I think that the most obvious answer to that question is, the information will be used in the operating room. I rose to mention that because I suspect that other people in the audience were thinking the same thing. 